\title{
Il percorso di continuità assistenziale ospedale- territorio nei pazienti con gravi cerebrolesioni acquisite. Le aspettative dei caregiver e dei professionisti
}

\author{
The path of continuity of care between hospital and territory in patients with severe brain \\ injury. The expectations of caregivers and professionals.
}

\author{
Raffaele Ferioli3 Monica Bolzani2 Maria Cristina Cornelli1 Lida Ghirardi1 Loris Guizzardi2
}

\author{
Roberta Onesti2 Antonella Dovani ${ }^{1}$ Andrea Davolo4 Giovanna Artioli1 Tiziana Mancini4
}

\begin{abstract}
RIASSUNTO
Utilizzando il modello teorico della GAP Analysis sono state analizzate le percezioni che gli operatori sanitari e i caregiver di pazienti "Gracer" (Gravi Cerebrolesioni Acquisite) hanno della continuità assistenziale (declinata in informativa, organizzativa e relazionale), per rilevare quali sono gli aspetti su cui il divario tra aspettative e realtà viene maggiormente sentito e quale è la dimensione della continuità assistenziale più importante sia per i professionisti della salute che per i caregiver. 122 professionisti della salute e 21 caregiver di pazienti Gracer ricoverati (fra 12 e 36 mesi dall'evento acuto) in quattro strutture sanitarie della regione Emilia-Romagna che utilizzavano e applicavano il Piano Assistenziale Individuale (PAI), hanno compilato un questionario riadattato dal ServQual per rilevare la percezione che i partecipanti avevano della continuità assistenziale informativa, organizzativa e relazionale effettiva (piano reale) ed attesa (piano ideale) nelle strutture sanitarie considerate. Per entrambi i gruppi la dimensione più importante della continuità assistenziale è risultata quella relazionale seguita con notevole scarto da quella informativa e da quella gestionale. I professionisti sanitari hanno descritto la realtà come peggiore delle aspettative in quasi tutte le dimensioni considerate. Per i caregiver la realtà è risultata peggiore delle aspettative soltanto per
\end{abstract} alcuni aspetti relativi alla continuità informativa e gestionale.

La ricerca ha messo in evidenza che la dimensione relazionale della continuità assistenziale andrebbe ulteriormente indagata, come confermato anche in letteratura. Occorrerebbe inoltre approfondire presso i professionisti l'area di insoddisfazione risultante dal bilancio negativo tra aspettative e realtà.

Parole chiave: Continuità assistenziale, caregiver, professionisti della salute, soddisfazione, lesioni cerebrali ABSTRACT

The present study analyses how continuity of care is perceived by health professionals and GRACER (Gravi Cerebrolesioni Acquisite Emilia Romagna) patients' caregivers, in order to investigate where the gap between expectations and reality is more heavily felt and which dimension of the continuity of care is the most important both for health professionals and GRACER patients' caregivers. The study has been developed following the Gap Analysis theoretical model. A questionnaire, based on ServQual model, was used to collect data about the three dimensions of the construct of continuity of care related to information, management and relation, declined along the lines of expectations and perception of reality. The questionnaire was administered to health professionals and caregivers of GRACER patients (12-36 months after the event) inside 4 healthcare institutes in Emilia Romagna. The PAI (Piano Assistenziale Individuale) approach was the methodology applied in these 4 sites.

To both groups the relational continuity was the most important dimension, followed at a long distance by the informational and the management ones. It has also been noted that to professionals reality is always worse than expectations, with the exception of only two items in the dimension of management continuity. To caregivers reality is worse than expectations in some items in the dimensions of information and management

The study has shown that the relational dimension of continuity of care should be more investigated, as confirmed by literature. More research is needed about the professionals dissatisfaction generated by the negative balance between expectations and perception of reality.

Key words: Continuity of patient care, caregiver, health professionals, expectations, perceptions, customer satisfaction, brain injury

1 Azienda Ospedaliero Universitaria di Parma

\section{Azienda USL di Parma}

3 Azienda Ospedaliero-Universitaria di Ferrara

4 Università degli Studi di Parma.

Correspondence to: Tiziana Mancini

Tel. 0521 034867; Fax 0521 034812;

e-mail tiziana.mancini@unipr.it

\section{INTRODUZIONE ALLO STUDIO}

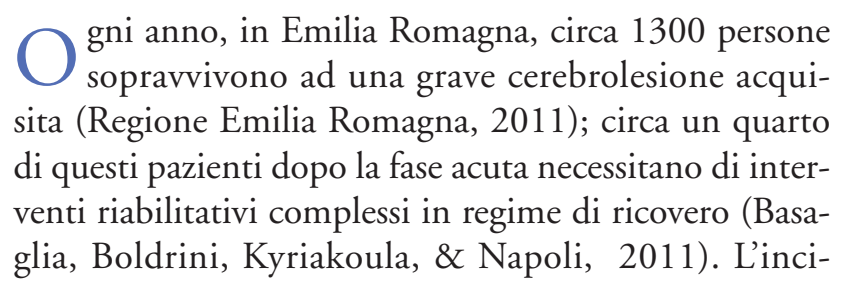


denza, l'alta numerosità e la complessità delle sequele disabilitanti, unite all'elevato impatto sociale e familiare, fanno dei pazienti Gracer (Grave Cerebrolesione acquisita) un problema sanitario e sociale di particolare rilevanza. Il livello di stress sperimentato dai familiari di pazienti con trauma cerebrale è tale che l'intervento professionale di supporto produce i suoi effetti perfino dopo 10-15 anni dall'evento traumatico (Verhaeghe et al., 2005).

La Regione Emilia Romagna ha predisposto un progetto specifico per le gravi cerebrolesioni acquisite. Esso prevede una rete riabilitativa Hub \& Spoke per garantire a questi pazienti tempestività nella presa in carico, adeguati livelli di cura e di continuità, equità nelle condizioni di accesso e di fruizione (Regione Emilia Romagna, 2011). Questo studio intende analizzare come la continuità assistenziale garantita a questi pazienti sia valutata sia dai caregiver che dai professionisti sanitari che lavorano all'interno di queste strutture.

Sono piuttosto recenti le prime revisioni sistematiche della letteratura volte ad individuare una definizione univoca del concetto di continuità assistenziale. La continuità assistenziale ha, infatti, assunto significati diversi nella medicina di base, nella salute mentale, in riferimento ai pazienti cronici o in condizioni specifiche. In tutte le discipline viene comunque riconosciuto che per una assistenza di alta qualità sono fondamentali tre tipi di continuità: quella informativa, quella gestionale (o longitudinale) e quella interpersonale o relazionale (Pandhi et al., 2006; Reid et al., 2002).

La continuità assistenziale può essere definita come un concetto multidimensionale che racchiude in sé due elementi fondamentali: una esperienza assistenziale vissuta dal paziente con il suo provider (tutti gli "attori" che forniscono servizi di salute) e una assistenza protratta nel tempo (Reid et al., 2002). Per essere considerata tale, la continuità assistenziale deve essere vissuta come connessa e coerente e deve comprendere in sé il significato di:

a) raccolta organizzata di informazioni mediche e sociali prontamente disponibili per qualsiasi operatore coinvolto nella cura (Continuità informativa);

b) erogazione di servizi tempestivi e puntuali all'interno di un piano di gestione condiviso (Continuità gestionale o organizzativa);

c) relazione continua tra paziente ed operatori caratterizzata dalla fiducia del paziente e dalla personale responsabilità dell'operatore. Il contatto prolungato tra professionista e paziente rappresenta il connettivo che collega l'assistenza nel tempo (Continuità interpersonale o relazionale; Saultz, 2003).

La mancanza di consenso del mondo scientifico non è limitata alla definizione concettuale, ma si estende anche agli strumenti utilizzati per misurare la conti- nuità: essi si sono concentrati per lo più sugli aspetti cronologici dell'assistenza, mentre più raramente anche sulla continuità informativa e interpersonale, misurata attraverso il numero di contatti con lo stesso medico nel tempo. Le misurazioni hanno inoltre sostanzialmente rispecchiato il concetto di continuità dal punto di vista dei professionisti più che quello dei pazienti (Freeman, 2005; Reid et al., 2002).

Viene generalmente riconosciuto che la continuità è un principio fondamentale nelle cure primarie (Pandhi \& Saultz, 2006 ; Reid et al., 2002), ma si conosce poco sulle percezioni della continuità dell'assistenza da parte dei pazienti (Saultz, 2003), per i quali risulta importante la continuità relazionale, soprattutto per quelli vulnerabili, come gli anziani (Brown, Mc William, \& Mai 1997; Nutting, Goodwin, Flocke, Zyzansky, \& Stange, 2003), i genitori di giovani pazienti, quelli con bassa scolarità, quelli con basso reddito (Nutting et al., 2003), le donne (Pereira \& Pearson, 2003). I pazienti con condizioni croniche valutano rilevante anche la trasmissione delle informazioni socio sanitarie (Pandhi \& Saultz, 2006). L'esigenza di discontinuità (avere un secondo parere, interagire con un nuovo medico per avere diverse prospettive) è stata documentata in pochi studi (Infante et al., 2004; Pandhi \& Saultz, 2006, Tarrant et al., 2003). I pazienti valutano positivamente avere lo stesso medico per la relazione che si instaura (Gabel et al., 1993; Lings et al., 2003; Perron \& Vannotti, 2004): nel tempo si acquisisce la confidenza per esprimere i propri bisogni, si impara a comprendersi (Infante et al., 2004); valutano inoltre positiva l'alleanza col medico per accordarsi sulla gestione del piano assistenziale (Gabel et al., 1993). L'idea che la confidenza e la fiducia in un medico si sviluppi nel tempo è suffragata da diversi studi qualitativi (Gabel et al., 1993; Infante et al., 2004; Tarrant et al., 2003). I pazienti che hanno malattie croniche o gravi danno particolare importanza alla continuità relazionale per ragioni di efficienza, non dovendo ripetere anamnesi complesse, di efficacia (la relazione crea maggiore coinvolgimento nell'assumere decisioni) e di fiducia che il loro medico si assumerà la responsabilità della loro assistenza (Pandhi et al., 2006; Guthrie et al., 2008).

Sia i pazienti che i familiari percepiscono molto carente la continuità assistenziale nella pianificazione pre-dimissione e nell'integrazione nella comunità (Rotondi et al., 2007).

Qualcuno si chiede se la continuità non sia solo un principio (forse datato) piuttosto che un attributo essenziale dell'assistenza (Christakis et al., 2004; Saultz $\&$ Lochner, 2005). Altri ritengono che a dispetto della sua scarsa considerazione in politica sanitaria la continuità relazionale rimane di vitale importanza (Guthrie et al., 2008; Saultz et al., 2004), soprattutto per i 
pazienti con problemi complessi (Nutting et al., 2003; Pandhi et al., 2006 ; Schers et al., 2002), sia perché è rilevante per i pazienti, sia perché facilita la continuità informativa e gestionale (Guthrie et al., 2008).

Il concetto di continuità assistenziale rimane quindi un costrutto poco chiaro nella letteratura. A questo si aggiunge il fatto che non ci sono riferimenti di cosa questa significhi per i pazienti Gracer.

\section{METODOLOGIA}

\section{Obiettivi}

L'obiettivo di questo studio è stato quello di analizzare la percezione che i caregiver di pazienti GRACER ed i professionisti che li assistono in alcune strutture sanitarie della Regione Emilia Romagna hanno della continuità assistenziale informativa, organizzativa e relazionale; più nello specifico rilevare, per i due gruppi, l'importanza relativa attribuita alle tre dimensioni delle continuità assistenziale e misurare il GAP percepito tra il piano delle pratiche (reale) e quello delle intenzioni (ideale).

\section{Contesto}

Sono stati contattati ed intervistati tutti i caregiver dei soggetti ricoverati nel maggio 2009, assistiti con applicazione del PAI (Legge 8 novembre 2000, n. 328; DPR n. 129 del 6 giugno 2001) presso le strutture sanitarie e servizi alla persona della Regione Emilia Romagna e che avevano avuto un evento traumatico da 12 a 36 mesi prima dell'intervista e tutti gli operatori delle medesime strutture contattate: gli Spoke T della provincia di Parma (Presidio di S. Secondo dell'AUSL di Parma, l'ASP di Langhirano e Colorno) e il Centro Hub dell'Azienda Ospedaliero - Universitaria di Ferrara. Le tre strutture con funzioni riabilitative diversificate, fanno parte del modello organizzativo a reti integrate di servizi a tipo "Hub \& Spoke" adottato per il progetto GRACER per la Regione Emilia Romagna.

\section{Strumenti e procedure}

Il disegno della ricerca, di tipo cross-sectional, ha utilizzato due questionari analoghi tesi a rilevare la soddisfazione che rispettivamente i careviger di pazienti Gracer e i professionisti sanitari hanno espresso nei confronti della continuità assistenziale garantita dalle strutture sanitarie considerate. Le due versioni del questionario sono state costruite sulla base del modello della Gap Analysis, ampiamente utilizzato nelle organizzazioni/servizi sanitari (Cuomo, 2000; Schiavi, 2004). Come è noto, questo modello, non considera la soddisfazione per un servizio come un valore assoluto, bensì come la diffe-

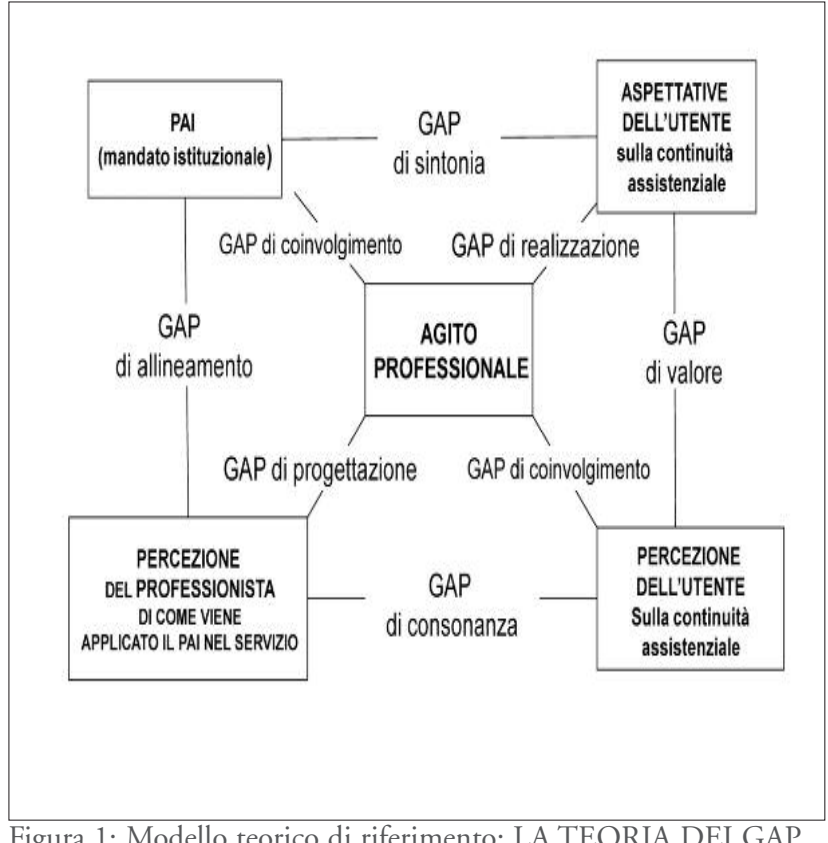

Figura 1: Modello teorico di riferimento: LA TEORIA DEI GAP.

renza che intercorre tra percezioni ed aspettative (Franci \& Corsi, 1998). Nella figura 1 è rappresentata l'operazionalizzazione dei costrutti del modello presi in considerazione in questo studio.

Per costruire gli item che nei questionari erano tesi a rilevare la soddisfazione percepita nei confronti della continuità assistenziale - declinata nei tre aspetti della continuità informativa, organizzativa e relazionale - i campi contenuti nelle schede utilizzate per la compilazione dei PAI dei pazienti GRACER sono stati sottoposti ad un'analisi del contenuto tematico finalizzata a ricavare una lista di dimensioni della continuità assistenziale comuni alle strutture sanitarie considerate. Tale lista è stata quindi sottoposta ad un gruppo di ricercatori che, partendo dalla definizione tridimensionale del costrutto di continuità assistenziale proposta da Saultz (2003), ha raggiunto l'accordo nella selezione 15 dimensioni, 5 per ognuno dei tre aspetti della continuità assistenziale. Ogni dimensione è stata poi declinata in item che, in accordo con le indicazioni fornite dal Modello del SERVQUAL (Zeithaml et al., 1991), fossero in grado di misurare nei caregiver come nei professionisti, sia le Aspettative (cosa si vorrebbe: PAI e Aspettative dell'utente nella fig. 1) che la Percezione del servizio (rispetto al momento dell'ultimo contatto: Percezione del professionista e Percezione dell'utente nella fig. 1). E' stata così costruita una scala in cui per ciascuno dei due piani di realtà considerati (Aspettative e Percezione del servizio), 5 item erano finalizzati a misurare la continuità informativa, 5 la continuità gestionale e 5 la continuità relazionale. L'attendibilità delle scale è stata misurata attraverso l'omogeneità interna misurata su tutti i partecipanti attraverso l'alpha di Cronbach (Tabella 1). 


\begin{tabular}{|c|c|c|}
\hline Aspetti della continuità & $\begin{array}{c}\text { Aspettative verso il } \\
\text { servizio }\end{array}$ & $\begin{array}{c}\text { Percezione del } \\
\text { servizio }\end{array}$ \\
\hline Informativa (5 item) & .67 & .74 \\
\hline Gestionale (5 item) & .45 & .55 \\
\hline Relazionale (5 item) & .68 & .79 \\
\hline
\end{tabular}

Tabella 1: Attendibilità ( $\alpha$ di Cronbach) degli indicatori di continuità assistenziale utilizzati nell'analisi dei dati.

Il confronto tra le due scale (Aspettative e Percezione del servizio) e tra i due punti di vista (Caregiver e Professionisti) è stato utilizzato per rilevare i quattro principali Gap del modello (Figura 1):

Il Gap di sintonia: misura la differenza tra le Aspettative dei Professionisti sanitari e quelle dei Caregiver;

- Il Gap di consonanza: misura la differenza tra le Percezioni dei Professionisti e quelle dei Caregiver; Il Gap di valore: misura differenza fra le Aspettative e la Percezione del servizio da parte del Caregiver;

- Il Gap di allineamento: misura la differenza tra le Aspettative e la Percezione del servizio da parte del Professionista.

In entrambe le versioni del questionario (quella per Caregiver e quella per Professionisti), ognuno dei 15 item è stato misurato su una scala Likert a 4 livelli (1 = per niente d'accordo; 4 = molto d'accordo). I questionari comprendevano anche un item a somma costante finalizzato a rilevare l'Importanza attribuita rispettivamente alle tre dimensioni della continuità assistenziale: informativa, gestionale, relazionale. I partecipanti dovevano in questo caso distribuire 100 punti tra le tre dimensioni della continuità assistenziale a seconda dell'importanza ad esse attribuita. Infine, sono stati rilevati dati socio-demografici.

Il questionario è stato sottoposto a 4 professionisti e 2 caregiver per verificarne la chiarezza e la durata di compilazione. Gli item sono risultati chiari e comprensibili; il tempo di compilazione è stato di circa 10 minuti per i professionisti e di 15/20 minuti per i caregiver.

Dopo aver ottenuto l'autorizzazione del Comitato Etico (Prot. N. 10614 del 02/04/2009) sono stati contattati i responsabili di struttura per concordare modalità e spazi per la somministrazione. Lo psicologo del centro ha informato i caregiver sulla possibilità di partecipare alla ricerca: tutti, ad eccezione di un caso, i caregiver dei pazienti che soddisfavano i criteri di campionamento hanno aderito alla ricerca. I responsabili delle strutture Spoke T e del Hub hanno contattato e consegnato il questionario a tutti i professionisti che, per il ruolo previsto nel PAI, garantiscono ai pazienti GRACER la continuità assistenziale: medico di struttura, fisiatra, neurologo, psicologo, infermiere, fisioterapista, logopedista, operatori socio sanitari della struttura. L'obiettivo della ricerca non era infatti quello di cogliere una particolare prospettiva, ma di analizzare quanto poteva emergere dall'insieme di tutti i punti di vista dei professionisti che operavano nelle strutture sanitarie considerate.

Il questionario è stato autocompilato dai professionisti e somministrato dall'intervistatore ai caregiver dei pazienti presenti nelle unità di cura considerate.

\section{Analisi dei dati}

Per l'elaborazione statistica dei dati è stato utilizzato il software SPSS (Statistical Package for Social Science) e data la differenza numerica dei due gruppi di partecipanti, l'analisi statistica è stata condotta con test non parametrici.

E' stato impiegato il test di Mann-Whitney per comparare le risposte fornite ad ogni item della scala dai due gruppi di partecipanti (Gap di sintonia e Gap di consonanza).

Per misurare lo scarto fra le Aspettative e la Percezione del servizio rispetto ai 15 aspetti della continuità assistenziale sui due gruppi di partecipanti (Gap di valore e Gap di allineamento) e la differenza fra l'importanza attribuita ai tre aspetti della continuità assistenziale è stato utilizzato il test di Wilcoxon. La profondità dei due GAP è stata misurata considerando la distribuzione dei partecipanti nei ranghi pari merito e no: essa ha permesso di definire come "insoddisfatti" quei professionisti/caregiver che percepiscono la realtà come inferiore alle aspettative, come "soddisfatti" quelli che, al contrario, hanno aspettative inferiori rispetto alla realtà; mentre sono stati definiti "in linea con le aspettative" i partecipanti le cui aspettative erano identiche alla percezione della realtà.

\section{RISULTATI}

\section{Partecipanti}

I caregiver a cui è stato somministrato il questionario sono stati individuati tra coloro che si prendevano cura del paziente (Centro di Formazione Albert Schweitzer, 2011). Sono stati complessivamente distribuiti e raccolti 21 questionari tutti compilati da "parenti" dei pazienti ricoverati. Di essi 12 hanno più di 50 anni, 9 sono uomini e 12 le donne; 11 lavorano e 10 non lavorano o lavorano in casa (Reid et al., 2002).

Tra i professionisti della salute ne sono stati contattati 162: di questi, 122 hanno risposto al questionario. Tra questi $4(3,3 \%)$ erano medici di struttura, 6 $(5,0 \%)$ fisiatri, $6(5,0 \%)$ psicologi, $44(36,7 \%)$ infermieri, $24(20,0 \%)$ fisioterapisti, $3(2,5 \%)$ logopedisti e $33(27,5 \%)$ operatori socio-sanitari (2 non hanno indicato la loro professione). Dei professionisti che hanno risposto al questionario l' $88,2 \%$ aveva meno 
di 50 anni; 21 erano maschi e 100 femmine. Il 60\% svolgeva la sua attività professionale presso la struttura in cui è stato contattato da meno di 5 anni, il $10 \%$ da più di 21 anni.

La distribuzione dei professionisti e dei caregiver in funzione struttura sanitaria in cui i questionari sono stati raccolti è presentata nella tabella 2 .

\begin{tabular}{|c|c|c|}
\hline Provenienza & Professionisti & Caregiver \\
\hline Spoke Colorno & $6(4,9 \%)$ & $1(4,8 \%)$ \\
\hline Spoke Langhirano & $20(16,4 \%)$ & $7(33,3 \%)$ \\
\hline Spoke San Secondo & $32(26,2 \%)$ & $1(4,8 \%)$ \\
\hline Hub Ferrara & $64(52,5 \%)$ & $12(57,1 \%)$ \\
\hline Totale & $122(100,0 \%)$ & $21(100,0 \%)$ \\
\hline
\end{tabular}

Tabella 2: Caratteristiche dei partecipanti in funzione della struttura e della tipologia di soggetto
Differenze nella percezione reale ed ideale della continuità assistenziale fra caregiver e professionisti: Gap di sintonia e Gap di consonanza.

In soltanto 3 delle 15 affermazioni le differenze tra Professionisti e Caregiver rispetto alle Aspettative nei confronti del servizio (Gap di sintonia) sono risultate statisticamente significative. I caregiver vorrebbero che il paziente fosse assistito dagli stessi operatori $(\mathrm{M}=3,67$ vs. 2,$61 ; \mathrm{p}=.000)$, che avesse un unico operatore come punto di riferimento per il percorso di cura $(\mathrm{M}=3,38$ vs. 2,$59 ; \mathrm{p}=.000$ ), che ci fosse collaborazione fra personale della struttura e personale dell'assistenza domiciliare $(\mathrm{M}=3,9$ vs. 3,$43 ; \mathrm{p}=.005)$ significativamente di più dei professionisti della salute. Sul piano reale, ossia delle Percezioni del servizio (Gap di consonanza) sono invece i Professionisti ad essere significativamente più d'accordo di quanto non lo siano i Caregiver con l'affermazione che la collaborazione fra personale della struttura e personale del territorio (e quindi la partecipazione di più figure) possa agevolare il percorso di cura dei pazienti Gracer $(\mathrm{M}=2,3$ vs. 2,$98 ; \mathrm{p}=.005$; tabella 3$)$.

\begin{tabular}{|c|c|c|c|c|c|}
\hline & & \multicolumn{2}{|c|}{ Aspettative verso il servizio } & \multicolumn{2}{|c|}{ Percezione del servizio } \\
\hline & & $\begin{array}{l}\text { Caregiver } \\
\text { M (SD) }\end{array}$ & $\begin{array}{l}\text { Professionisti M } \\
\text { (SD) }\end{array}$ & $\begin{array}{l}\text { Caregiver } \\
\text { M (SD) }\end{array}$ & $\begin{array}{l}\text { Professionisti M } \\
\text { (SD) }\end{array}$ \\
\hline \multirow{5}{*}{ 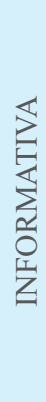 } & $\begin{array}{l}\text { 1-16. Distribuzione di materiale informativo sul percorso di cura (piano } \\
\text { assistenziale individuale, consenso al trattamento dei dati, consenso } \\
\text { informato, regolamento della struttura di ricovero) }\end{array}$ & $3,76(0,54)$ & $3,76(0,54)$ & $3,05(0,80)$ & $3,28(0,80)$ \\
\hline & $\begin{array}{c}\text { 4-19. Scambio di informazioni cliniche e assistenziali dei pazienti quan- } \\
\text { do questi vengono trasferiti da un servizio all'altro }\end{array}$ & $3,76(0,44)$ & $3,79(0,77)$ & $3,20(0,70)$ & $3,06(0,82)$ \\
\hline & $\begin{array}{l}\text { 7-22. Informazioni al familiare o chi assiste il paziente sugli ausili utiliz- } \\
\text { zabili per favorire il recupero e la massima autonomia dell'assistito }\end{array}$ & $4,00(0,00)$ & $3,71(0,52)$ & $3(0,95)$ & $3,38(0,70)$ \\
\hline & $\begin{array}{l}\text { 10-25. Informazioni sulle barriere architettoniche presenti al domicilio } \\
\text { dell' assistito }\end{array}$ & $3,67(0,80)$ & $3,46(0,81)$ & $2,62(1,12)$ & $3,27(0,89)$ \\
\hline & $\begin{array}{c}\text { 13-28. Informazioni al familiare o chi assiste il paziente sui servizi socio } \\
\text { sanitari presenti nel territorio }\end{array}$ & $3,95(0,22)$ & $3,66(0,63)$ & $2,76(1,09)$ & $3,25(0,83)$ \\
\hline \multirow{5}{*}{ 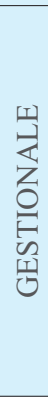 } & $\begin{array}{l}\text { 2-17. Dovrebbero essere sempre gli stessi operatori ad assistere lo } \\
\text { stesso paziente }\end{array}$ & $3,67(0,66)$ & $2,61 * * *(0,93)$ & $3,33(0,80)$ & $3,02(0,89)$ \\
\hline & $\begin{array}{l}\text { 3-18. II familiare o chi assiste il paziente dovrebbe poter trovare gruppi } \\
\text { di sostegno formati di persone che hanno vissuto o vivono la stessa } \\
\text { loro esperienza }\end{array}$ & $3,29(0,85)$ & $3,38(0,71)$ & $2,90(1,04)$ & $3,16(0,82)$ \\
\hline & $\begin{array}{l}\text { 5-20. II medico di medicina generale dovrebbe partecipare alle decisio- } \\
\text { ni sui percorsi di cura dei suoi pazienti assistiti nel reparto }\end{array}$ & $3,62(0,74)$ & $3,24(0,83)$ & $2,30(1,22)$ & $2,81(0,96)$ \\
\hline & $\begin{array}{l}\text { 8-23. II familiare o chi assiste il paziente dovrebbe essere coinvolto dal } \\
\text { personale sanitario sulle decisioni che riguardano il percorso di cura del } \\
\text { proprio assistito }\end{array}$ & $3,76(0,44)$ & $3,50(0,72)$ & $3,24(0,70)$ & $3,09(0,84)$ \\
\hline & $\begin{array}{c}\begin{array}{c}\text { 11-26. II paziente dovrebbe avere un unico operatore come referente } \\
\text { del percorso di cura }\end{array} \\
\text {. }\end{array}$ & $3,38(0,97)$ & $2,59 * * *(0,95)$ & $2,86(0,85)$ & $2,53(0,87)$ \\
\hline \multirow{5}{*}{ 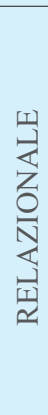 } & $\begin{array}{l}\text { 14-29. Quando il percorso di cura sta per concludersi, il personale sani- } \\
\text { tario dovrebbe collaborare con il personale dell'assistenza domiciliare } \\
\text { territoriale }\end{array}$ & $3,90(0,44)$ & $3,43 * *(0,77)$ & $2,30(1,03)$ & $2,98^{* *}(0,88)$ \\
\hline & $\begin{array}{l}\text { 6-21. II familiare o chi assiste il paziente dovrebbe avere la possibilità di } \\
\text { parlare con un professionista qualora si senta giù di morale }\end{array}$ & $3,71(0,72)$ & $3,74(0,46)$ & $3,14(1,06)$ & $3,21(0,83)$ \\
\hline & $\begin{array}{l}\text { 9-24. II familiare o chi assiste il paziente dovrebbe essere ascoltato dal } \\
\text { medico di riferimento }\end{array}$ & $3,86(0,36)$ & $3,69(0,48)$ & $3,38(0,80)$ & $3,31(0,86)$ \\
\hline & $\begin{array}{l}\text { 12-27. In un centro come questo il personale sanitario dovrebbe utiliz- } \\
\text { zare con i familiari o con chi assiste il paziente una terminologia chiara } \\
\text { e comprensibile }\end{array}$ & $3,95(0,22)$ & $3,83(0,44)$ & $3,62(0,59)$ & $3,53(0,68)$ \\
\hline & $\begin{array}{c}\text { 15-30. Il personale sanitario dovrebbe aggiornare costantemente il } \\
\text { familiare o chi assiste il paziente sull'andamento del piano assistenziale }\end{array}$ & $3,90(0,30)$ & $3,60(0,56)$ & $3,24(0,77)$ & $3,16(0,65)$ \\
\hline
\end{tabular}

Tabella 3 : Differenza tra Caregiver e Professionisti (Gap di sintonia e Gap di consonanza) rispetto alle tre dimensioni della continuità assistenziale (valori medi, deviazioni standard e confronti Mann-Whitney sui 15 item delle due scale) 
$\mathrm{Al}$ di là delle differenze che sono risultate statisticamente significative, le analisi descrittive lasciano emergere una più ampia variabilità delle risposte sulla Percezione del servizio da parte dei caregiver che dei professionisti.

Differenze fra aspettative e realtà rispetto alle diverse dimensioni della "continuità assistenziale": Gap di valore e Gap di allineamento

Confrontando per entrambi i gruppi di partecipanti i due piani di realtà, emergono differenze statisticamente significative per molti dei 15 item della scala. In generale, la Percezione del servizio è risultata sia per i Caregiver (Gap di valore) che per i Professionisti (Gap di allineamento) come inferiore alle Aspettative.

Per i Professionisti il confronto tra Aspettative e Percezione del servizio ha fatto emergere differenze statisticamente significative (test di di Wilcoxon) su 13 dei 15 item considerati.

La tabella 4 presenta anche la profondità del gap rilevato: come si può notare, se dai 4 ai 6 professionisti su 10 hanno dichiarato di avere aspettative in linea con la loro percezione del servizio, rilevante risulta comunque la percentuale di quanti hanno dichiarato di percepire la realtà come inferiore alle aspettative. La situazione più grave riguarda il gap rispetto alla continuità informativa e gestionale in cui le percentuali di professionisti "insoddisfatti" si attestano mediamente attorno al $40 \%$ nel primo caso e al $38 \%$ nel secondo. Al contrario più alta è risultata la percentuale di professionisti che si sono dichiarati "soddisfatti" di come nelle rispettive strutture di appartenenza viene gestita la continuità gestionale (mediamente pari al 20\%).Per i caregiver è soprattutto rispetto alla continuità informativa che la realtà viene considerata peggiore delle aspettative (Tabella 5). Il servizio viene percepito come significativamente peggiore delle loro aspettative sul versante della continuità informativa, ma solo alcuni aspetti della continuità gestionale e relazionale lasciano emergere un gap statisticamente significativo.

Essi riguardano la partecipazione del medico di medicina generale alle decisioni sui percorsi di cura dei pazienti assistiti nel reparto nel caso della continuità gestionale e la collaborazione del personale sanitario con il personale dell'assistenza domiciliare territoriale, nonché la volontà di aggiornare costantemente il familiare o chi assiste il paziente sull'andamento del piano assistenziale nel caso della continuità relazionale.

Osservando il gap rilevato (tabella 5) si può evidenziare che gli "insoddisfatti" sono in questo caso in numero maggiore dei Caregiver "in linea con le aspettative" su tutte e tre le dimensioni della continuità assistenziale. La situazione più grave riguarda ancora una volta il gap rispetto alla continuità informativa dove i caregiver "insoddisfatti" sono mediamente circa 6 su 10, mentre quelli "insoddisfatti" rispetto alla continuità relazionale e gestionale sono mediamente la metà. E' in riferimento alla continuità gestionale che è stato registrato il numero più elevato di caregiver "soddisfatti".

\begin{tabular}{|c|c|c|c|c|c|c|c|}
\hline & \multirow{3}{*}{$\begin{array}{c}\text { Confronto } \\
\text { Aspettative/Percezion } \\
\text { e del servizio (test di } \\
\text { Wilcoxon) }\end{array}$} & \multicolumn{6}{|c|}{ Profondità del Gap } \\
\hline & & \multicolumn{2}{|c|}{ Insoddisfatti } & \multicolumn{2}{|c|}{ Soddisfatti } & \multicolumn{2}{|c|}{ In linea con le aspettative } \\
\hline & & $\mathrm{N}$ & $\%$ & $\mathrm{~N}$ & $\%$ & $\mathrm{~N}$ & $\%$ \\
\hline \multirow{5}{*}{$\begin{array}{l}\text { Continuità } \\
\text { informativa }\end{array}$} & $16-1 * * *$ & 51 & 42.15 & 7 & 5.79 & 63 & 52.07 \\
\hline & $19-4 * * *$ & 69 & 57.02 & 3 & 2.48 & 49 & 40.5 \\
\hline & $22-7 * * *$ & 40 & 33.06 & 8 & 6.61 & 73 & 60.33 \\
\hline & $25-10 *$ & 34 & 28.1 & 14 & 11.57 & 73 & 60.33 \\
\hline & $28-13 * * *$ & 47 & 38.84 & 10 & 8.26 & 62 & 51.24 \\
\hline \multirow{4}{*}{$\begin{array}{l}\text { Continuità } \\
\text { gestionale }\end{array}$} & $17-2 * * *$ & 14 & 11.57 & 48 & 39.67 & 59 & 48.76 \\
\hline & $18-3$ & 42 & 34.71 & 22 & 18.18 & 57 & 47.11 \\
\hline & $20-5^{* * *}$ & 52 & 42.98 & 12 & 9.92 & 57 & 47.11 \\
\hline & $23-8 * * *$ & 53 & 43.8 & 18 & 14.88 & 50 & 41.32 \\
\hline \multirow{5}{*}{$\begin{array}{l}\text { Continuità } \\
\text { relazionale }\end{array}$} & $29-14 * * *$ & 53 & 43.8 & 17 & 14.05 & 52 & 42.98 \\
\hline & $21-6 * * *$ & 49 & 40.5 & 5 & 4.13 & 65 & 53.72 \\
\hline & $24-9 * * *$ & 46 & 38.02 & 13 & 10.74 & 62 & 51.24 \\
\hline & $27-12 * * *$ & 34 & 28.1 & 7 & 5.79 & 79 & 65.29 \\
\hline & $30-15^{* * *}$ & 48 & 39.67 & 11 & 9.09 & 61 & 50.41 \\
\hline
\end{tabular}

Tabella 4: Gap di Allineamento: numero di Professionisti Soddisfatti, Insoddisfatti e In linea con le aspettative (valori assoluti, N. 122) 


\begin{tabular}{|c|c|c|c|c|c|c|c|}
\hline & \multirow{3}{*}{$\begin{array}{l}\text { Confronto } \\
\text { Aspettative/ } \\
\text { Percezione del } \\
\text { servizio (test di } \\
\text { Wilcoxon) }\end{array}$} & \multicolumn{6}{|c|}{ Profondità del Gap } \\
\hline & & \multicolumn{2}{|c|}{ Insoddisfatti } & \multicolumn{2}{|c|}{ Soddisfatti } & \multicolumn{2}{|c|}{ In linea con le aspettative } \\
\hline & & N & $\%$ & $\mathrm{~N}$ & $\%$ & N & $\%$ \\
\hline \multirow{5}{*}{$\begin{array}{l}\text { Continuità } \\
\text { informativa }\end{array}$} & $16-1^{*}$ & 11 & 52.38 & 1 & 4.76 & 9 & 45 \\
\hline & $19-4 *$ & 12 & 60 & 1 & 5 & 7 & 33.33 \\
\hline & $22-7^{* * *}$ & 13 & 61.9 & 0 & 0 & 8 & 38.1 \\
\hline & $25-10^{* * *}$ & 12 & 57.14 & 0 & 0 & 9 & 42.86 \\
\hline & $28-13 * * *$ & 13 & 61.9 & 0 & 0 & 8 & 38.1 \\
\hline \multirow{5}{*}{$\begin{array}{l}\text { Continuità } \\
\text { gestionale }\end{array}$} & $17-2$ & 6 & 28.57 & 1 & 4.76 & 14 & 66.67 \\
\hline & $18-3$ & 10 & 47.62 & 5 & 23.81 & 6 & 30 \\
\hline & $20-5 * * *$ & 12 & 60 & 0 & 0 & 8 & 38.1 \\
\hline & $23-8$ & 12 & 57.14 & 3 & 14.29 & 6 & 28.57 \\
\hline & $26-11$ & 10 & 47.62 & 2 & 9.52 & 9 & 45 \\
\hline \multirow{5}{*}{$\begin{array}{l}\text { Continuità } \\
\text { relazionale }\end{array}$} & $29-14 * * *$ & 17 & 85 & 1 & 5 & 2 & 9.52 \\
\hline & $21-6$ & 9 & 42.86 & 2 & 9.52 & 10 & 47.62 \\
\hline & $24-9$ & 7 & 33.33 & 0 & 0 & 14 & 66.67 \\
\hline & $27-12$ & 6 & 28.57 & 0 & 0 & 15 & 71.43 \\
\hline & $30-15 * * *$ & 10 & 47.62 & 0 & 0 & 11 & 9.09 \\
\hline
\end{tabular}

Tabella 5: Gap di valore: numero dei Caregiver Soddisfatti, Insoddisfatti e In linea con le aspettative (valori assoluti, N. 21)

Importanza delle tre dimensioni del costrutto "continuità assistenziale"

Ma quale è l'importanza che Professionisti e Caregiver attribuiscono alle tre dimensioni della continuità assistenziale?

I confronti statistici hanno evidenziato che la dimensione considerata più importante da entrambi i gruppi di partecipanti è quella relazionale che nel caso dei professionisti $(\mathrm{M}=44,43)$ si differenzia significativamente da quella informativa $(\mathrm{M}=28,49)$ e gestionale $(\mathrm{M}=26,81$; rispettivamente $\mathrm{p}<.001$ e $\mathrm{p}<.001)$ e nel caso dei Caregiver $(M=38,81)$ soltanto da quella informativa $(M=27,17 ; \mathrm{p}<.05)$.

\section{DISCUSSIONE}

Partendo da una definizione multidimensionale della continuità assistenziale (Pandhi et al., 2006; Reid et al., 2002; Saultz, 2003 ), l'obiettivo di questo studio è stato quello di rilevare la percezione che i professionisti della salute e gli utenti di alcune strutture sanitarie per pazienti Gracer dell'Emilia Romagna hanno rispetto a come dovrebbe essere gestita e a come viene concretamente gestita la continuità relazionale, informativa e gestionale nei servizi considerati. Per raggiungere tale obiettivo, facendo riferimento al modello teorico della Gap Analysis e alle indicazioni metodologiche presenti in letteratura (Cuomo, 2000; Zeithaml et al., 1991), è stato costruito un questionario che, considerando il possibile scarto esistente tra le loro aspettative e le loro percezioni di realtà, ha permesso di rilevare i livelli di in/soddisfazione dei professionisti e dei caregiver nei confronti del servizio, nonché l'importanza da essi attribuita alle tre diverse dimensioni della continuità assistenziale. In sintonia con gli indirizzi regionali riferiti alla necessità di avvicinare quanto più possibile i servizi alle esigenze dell'utenza, potenziando la capacità di ascolto e di relazione con i cittadini anche attraverso la valutazione delle aspettative e della soddisfazione dei pazienti (Agenzia Sanitaria regionale, 2003), la ricerca qui presentata intendeva anche suggerire possibili misure di rimedio e di correzione della continuità assistenziale nei servizi considerati.

I risultati hanno evidenziato che, rispetto alle tre dimensioni della continuità assistenziale, è quella relazionale ad essere considerata sia dai professionisti che dai caregiver come più importante delle altre due dimensioni della continuità. Lo studio conferma quindi quanto per i caregiver le esigenze di continuità assistenziale facciano soprattutto riferimento alla garanzia dell'assistenza nel tempo e alla possibilità di avere persone e punti di riferimento stabili.

Tuttavia, come indica anche la letteratura (Green, 2005; Pandhi \& Saultz, 2006), i caregiver di questi pazienti che presentano gravi problemi di salute, valutano importante anche la continuità gestionale rispetto alla quale, diversamente dai professionisti, non percepiscono forti discrepanze tra aspettative e realtà. E' 
invece la continuità informativa a generare nei caregiver, come nei professionisti, maggiore insoddisfazione: essi ritengono, infatti, che la distribuzione e lo scambio di informazioni relative alla situazione dei propri parenti siano inferiori rispetto a quanto dovrebbero essere. Tra gli ambiti riferiti alla continuità relazionale emerge un'area di forte insoddisfazione per i caregiver: confermando quanto già è emerso in letteratura (Rotondi et al., 2007) è quella legata al post-dimissioni rispetto cui essi avvertono forte la necessità di migliorare i rapporti di collaborazione tra personale sanitario e personale dell'assistenza domiciliare.

Dai risultati emerge, quindi, un'ampia area di insoddisfazione. Essa è stata espressa da entrambe le categorie di partecipanti alla ricerca, seppure sia risultata più evidente per i caregiver che per i professionisti. Sebbene all'interno di queste strutture si utilizzi il PAI come strumento di lavoro che valorizza e promuove il contributo professionale del singolo, benché siano previsti momenti di formazione sul campo quali preziosi momenti di confronto e di scambio, e nonostante il numero dei pazienti Gracer e la loro permanenza sia tale da permettere a ogni componente dell'équipe di costruire relazioni e stabilire rapporti, il bilancio fra aspettative e realtà è sostanzialmente negativo. Questa percezione negativa potrebbe facilitare l'esposizione dei professionisti che svolgono una professione "hightouch" al rischio di burnout e dei caregiver a un eccessivo burden.

Rispetto a quanto già presente in letteratura a questo studio vanno riconosciuti alcuni meriti. In primo luogo quello di avere affrontato il tema della valutazione della continuità assistenziale prendendo in considerazione e confrontando, seppure su gruppi molto limitati numericamente, il punto di vista dei caregiver con quello dei professionisti sanitari. A questo pregio si aggiunge anche quello di avere affrontato la questione della continuità assistenziale prendendo a riferimento una categoria di utenti, i pazienti Gracer che, richiedendo complessi interventi riabilitativi e di supporto, mettono forse più di altre patologie in gioco il problema della continuità assistenziale. Infine, nel confermare l'importanza di considerare le diverse dimensioni della continuità assistenziale (relazionale, informativa e gestionale), le diverse dimensioni temporali (aspettative e realtà) e i diversi punti di vista coinvolti nel processo della sua misurazione (operatori e caregiver), la ricerca ha anche permesso di costruire uno strumento di misurazione che potrebbe rivelarsi utile anche in altri contesti sanitari. La costruzione ad hoc di questo strumento costituisce tuttavia al tempo stesso un limite della ricerca, accanto a quello della scarsa numerosità dei partecipanti allo studio e soprattutto dei caregiver. Seppure questo limite deriva dalle scelte metodologiche effettuate e dei contesti in cui la ricerca è stata effettuata, esso limita, di fatto, da un lato la possibilità di confrontare i livelli di soddisfazione o insoddisfazione per la continuità assistenziale espressi dalle diverse figure professionali che sono state considerate e/o le differenze rilevate rispetto alle diverse strutture che sono state oggetto della rilevazione, dall'altro la generalizzazione dei risultati ottenuti ad altre realtà operative regionali o italiane.

A questo limite aggiungiamo anche quelli che la letteratura evidenzia rispetto ai possibili utilizzi della teoria dei Gap e alle sue applicazioni pratiche (Zeithaml et al., 1991). Nonostante questi limiti pensiamo tuttavia che i risultati conseguiti forniscano chiare indicazioni su cosa sia possibile iniziare a fare per costruire servizi e strutture dedicate ai pazienti Gracer più in linea con i bisogni non solo dell'utenza, ma anche dei professionisti che in esse lavorano.

\section{CONCLUSIONI}

In conclusione, i risultati della ricerca hanno messo in evidenza come i Gap di allineamento e di valore siano molto più profondi di quelli di sintonia e di consonanza sulla quale professionisti e caregiver tendono ad allineare maggiormente le loro percezioni.

Rispetto ai gap di sintonia, ci sembra interessante evidenziare, ai fini di auspicabili miglioramenti dei servizi, l'importanza che i caregiver più dei professionisti hanno attribuito ad alcuni aspetti della continuità gestionale che andrebbero a loro avviso potenziati: il fatto di avere gli stessi operatori ad assistere lo stesso paziente, di avere un unico operatore come referente del percorso di cura e la collaborazione fra personale della struttura e del territorio quando il percorso di cura sta per concludersi. E' proprio su quest' ultimo aspetto che le percezioni dei professionisti e dei caregiver rispetto a quanto viene effettivamente fatto non concordano: i professionisti si ritengono, infatti, più soddisfatti di quanto non lo siano i caregiver dei pazienti che hanno in carico.

Le discrepanze che questa ricerca ha evidenziato suggeriscono quindi, in conclusione, un impegno e uno sforzo costante da parte delle istituzioni per rendere il servizio più allineato alle aspettative dell'utenza, soprattutto rispetto agli aspetti gestionali ed organizzativi.

Sempre sul versante più prettamente applicativo, la ricerca ha di per se avuto anche la funzione di dare voce attraverso la dimensione dell'ascolto empatico ai caregiver che vi hanno partecipato. Ne è una dimostrazione la soddisfazione espressa dai caregiver contattati per il fatto che il loro parere fosse ritenuto oggetto di ricerca e che il loro apporto fosse considerato importante quanto quello dei professionisti. 


\section{BIBLIOGRAFIA}

Agenzia Sanitaria Regionale (2003). Servizi sanitari e cittadini: segnali e messaggi. Regione Emilia Romagna, Dossier 85.

Basaglia, N., Boldrini, P., Kyriakoula, P., \& Napoli, N. (2011). Quante sono le persone che sopravvivono a una grave cerebrolesione acquisita? Risultati di due indagini relative all'anno 2001 svolte presso le strutture sanitarie della regione Emilia Romagna. Data accesso 2 maggio, 2011, da http://www.gracer.it/.

Brown, J.B., Mc William, C.L., \& Mai, V. (1997). Barriers and facilitators to seniors' independence. Perceptions of seniors, caregivers, and health care providers. Canadian Family Physicians, 43, 469-75.

Centro di Formazione Albert Schweitzer. Stress e sindrome del burnout tra gli operatori della sanità. Data accesso 2 maggio, 2011, da http://www.gracer.it/; http://www.cfschweitzer.org/news/corsi2004/abstractsi ntesiburnout.pdf .

Christakis, D.A., Kazak, A.E., Wright, J.A., Zimmerman, F.J., Basset, L., \& Connel, F.A. (2004). What factors are associated with achieving high continuity of care? Family Medicine, 36, 55-60.

Cuomo, M.T. (2000). La customer satisfaction: vantaggio competitivo e creazione di valore. Padova: Cedam.

Decreto del Presidente del Consiglio dei Ministri. Atto di indirizzo e coordinamento in materia di prestazioni socio-sanitarie. Gazzetta Ufficiale n. 129 del 6 giugno 2001, articolo 2, comma1, comma 2.

Franci, A., \& Corsi, M. (1998). Verso l'assistenza domiciliare integrata: una proposta di metodo per valutare la qualità percepita. Padova: Edizioni Summa.

Freeman, GK. (2005). Still not quite there with definition? but the challange is to measure "interpersonal" and show it makes a difference [letter]. Data accesso 24 maggio, 2011, da http://www.annfammed.org/ cgi/eletters/3/2/159\#1699.

Gabel, L.L., Lucas, J.B., \& Westbury, R.C. (1993). Why do patients continue to see the same physician? Family Practice Research Journal, 13, 133-47.

Green, LA. (2005). Reframing continuity [eletter]. Data accesso 21 maggio, 2011, da http://annfammed.org.cgi/eletters/3/2/159\#1682, 9.

Guthrie, B., Saultz, J.W., Freeman, G.K., \& Haggerty, J.L. (2008). Continuity of care matters, British Medical Journal, 337, 867.

Infante, F.A., Proudfoot, J.G., Powell, D.G., Bubner, T.K., Holton, C.H., Beilby, J.J., \& Harris, M.F. (2004). How people with chronic illnesses view their care in general practice: a qualitative study. The Medical Journal of Australia, 181, 70-3.

Legge 8 novembre 2000, n. 328. Legge quadro per la realizzazione del sistema integrato di interventi e servizi sociali. Gazzetta Ufficiale n. 265 del 13 novembre
2000-Supplemento ordinario n. 186.

Lings, P., Evans, P., Seamark, D., Seamark, C., Sweeney, K., \& Dixon, M.G. (2003). The doctor-patient relationship in US primary care. Journal of Reality Society of Medicine, 96,180-4.

Nutting, P.A., Goodwin, M.A., Flocke, S.A., Zyzansky, S.J., Stange, K.C. (2003). Continuity of primary care: to whom does it matter and when? The Annual of Family Medicine, 1,149-55.

Pandhi, N., \& Saultz, JW. (2006). Patients' perceptions of interpersonal continuity of care. The Journal of the American Board of Family Medicine, 19, 390-397.

Pereira, A.G., \& Pearson, S.D. (2003). Patient attitudes toward continuity of care. Archives of Internal Medicine, 163, 909-12.

Perron, N.J., \& Vannotti, M. (2004). Patients who attend a private practice vs. a university outpatient clinic: how do they differ ? Swiss Medical Weekly, 134, 730-7.

Regione Emilia Romagna. IL PROGETTO GRACER: la rete riabilitativa Hub\& Spoke per le gravi cerebrolesioni acquisite. Data accesso 2 maggio, 2011, da http://www.gracer.it/.

Reid, R., Haggerty, J., \& Mc Kendry, R. (2002). Defusing the confusion: concepts and measures of continuity of healthcare, final report. Canadian Health Services Foundation.

Rotondi, A.J., Sinkule, J., Balzer, K., Harris, J., \& Moldovan, R. (2007). A qualitative needs assessment of persons who have experienced traumatic brain injury and their primary family caregivers. The Journal of Head Trauma Rehabilitation, 22 (1),14-25.

Saultz, JW. (2003). Defining and measuring interpersonal continuity of care. The Annals of Family Medicine, 1, 134-143.

Saultz, J.W., \& Albedaiwi, W. (2004). Interpersonal continuity of care and patient satisfaction: a critical rewiew. The Annals of Family Medicine, 2, 445-1.

Saultz, J.W., \& Lochner, J. (2005). Continuità interpersonale dell'assistenza e risultati assistenziali: una revisione critica. The Annals of Family Medicine, 3, 159-166.

Schers, H., Webster, S., van den Hoogen, H., Avery, A., Grol, R.,\& Van den Bosch, W. (2002). Continuity of care in general practice: a survey of patients' view. British Journal of General Practice, 52, 459.

Schiavi, G. (2004). La misurazione della Customer Satisfaction nelle aziende sanitarie. Milano: Franco Angeli.

Tarrant, C., Windridge, K., Beulton, M., Baker, R., \& Freeman, G.(2003). How important is personal care in general practice? British Medical Journal, 326, 1310.

Verhaeghe, S., Defloor, T., \& Grypdonck, M. (2005). Stress and coping among families of patients with traumatic brain injury: a review of the literature. Journal of Clinical Nursing, 14, $1004-1012$.

Zeithaml, V.A., Parasuraman, A., \& Berry, L. (1991). Servire qualità. Milano: McGraw-Hill. 